\title{
Joint versus separate inpatient rehabilitation treatment for patients with alcohol use disorder or drug use disorder: an observational study
}

This article was published in the following Dove Press journal: Substance Abuse and Rehabilitation

\author{
Stefan Bender ${ }^{1}$ \\ Michael Specka ${ }^{2}$ \\ Angela Buchholz ${ }^{3}$ \\ Stefan Hölscher ${ }^{2}$ \\ Fred Rist ${ }^{4}$ \\ Thomas W Heinz ${ }^{5,6}$ \\ Fabrizio Schifano ${ }^{7}$ \\ Norbert Scherbaum ${ }^{2}$ \\ 'Psychiatry, Psychotherapy and \\ Psychosomatics, LWL-Hospital \\ Marsberg, Marsberg, Germany; \\ ${ }^{2}$ Department of Addictive Behaviour \\ and Addiction Medicine, LVR- \\ Hospital Essen, Faculty of Medicine, \\ University of Duisburg-Essen, Essen, \\ Germany; ${ }^{3}$ nstitute of Medical \\ Psychology; University Hospital \\ Hamburg-Eppendorf, Hamburg, \\ Germany; ${ }^{4}$ Insitute for Psychology, \\ University of Münster, Münster, \\ Germany; ${ }^{5}$ Psychiatry, Psychotherapy \\ and Psychosomatics, LWL-Hospital \\ Warstein, Warstein, Germany; \\ ${ }^{6}$ Addiction Hospital St. Vitus, Visbek, \\ Germany; ${ }^{7}$ Psychopharmacology, \\ Drug Misuse and Novel Psychoactive \\ Substances Research Unit, School \\ of Life and Medical Sciences, \\ University of Hertfordshire, Hatfield, \\ Hertfordshire, UK
}

Correspondence: Norbert Scherbaum Department of Addictive Behavior and Addiction Medicine, LVR-Hospital Essen, Faculty of Medicine, University of Duisburg-Essen, Virchowstraße 174, 44I47 Essen, Germany

Tel +492017227202

Fax +49 20I 7227254

Email norbert.scherbaum@uni-due.de
Background: In many national treatment systems, patients with alcohol use disorders (AUD) and those with drug use disorders (DUD) are treated separately, while other systems provide joint treatment for both kinds of substance use disorders (SUDs). Regarding long-term rehabilitation treatment of DUD and AUD patients, there is however a lack of empirical studies on the comparison between a separate versus joint treatment modality.

Methods: Data were gathered from 2 rehabilitation units located in small towns from the same German region. One unit provided treatment to a mixed group of AUD and DUD patients, while the other unit treated the 2 groups separately. Staffing, funding, and treatment programs were otherwise similar between facilities. Data were gathered from standardized routine documentation and standardized interviews. In order to understand correlates of premature treatment termination, a logistic regression analysis was performed, with treatment modality and type of SUD as main predictors, and a range of patient characteristics as covariates.

Results: Patients (N=319) were diagnosed with AUD (48\%), DUD (34\%), or AUD plus DUD $(18 \%)$. Patients in joint treatment showed a higher prevalence of lapses during treatment than those in separate treatment ( $26 \%$ versus $12 \% ; p=0.009)$, but there was no significant difference in the prevalence of premature terminations ( $38 \%$ versus $44 \%, p=0.26)$. Treatment modality and interaction between modality and type of SUD was not significantly associated with premature termination. Joint treatment completers showed higher satisfaction with treatment than separate treatment completers $(p<0.001)$.

Conclusion: We found no evidence here for a difference between treatment modalities in terms of premature termination rate. Satisfaction level was higher in those who completed joint treatment compared to separate treatment.

Keywords: alcohol use disorder, drug use disorder, rehabilitation treatment, inpatient treatment, joint treatment, long-term treatment

\section{Introduction}

National treatment systems differ markedly in terms of integration of services provided to subjects with an alcohol use disorder (AUD), on the one hand, and those with drug use disorders (DUD, including illicit psychotropic substances such as opiates, cocaine, cannabis, or amphetamines), on the other hand. For example, in the Netherlands, People's Republic of China, Canada, or Finland, care for AUD and DUD is highly integrated in terms of funding, organization, and professionals involved. ${ }^{1}$ In other countries, such as Switzerland, Poland, England, France, or Germany, treatment systems have traditionally been much more separated. Moreover, even a high degree 
of integration does not necessarily mean that patients are treated together, within the same facility and with the same treatment program. ${ }^{1}$

In many countries, the long-term inpatient rehabilitation ("Rehab") treatment constitutes a typical component of the substance use disorder (SUD; including both AUD and DUD) treatment package. The long-term (several weeks to months), intensive, highly structured Rehab approach aims at treating a group/community of patients, helping them in both maintaining abstinence and achieving a drug-/alcohol-free lifestyle. In some countries, such as the USA, Australia or the United Kingdom, many Rehab providers offer the same treatment package for all SUDs. In other countries, Rehab treatments for AUD and DUD patients are traditionally separated. ${ }^{2}$

In Germany, medical inpatient rehabilitation treatment for AUD patients has been available since the 19th century. ${ }^{3}$

After a sharp increase in psychotropic drug use during the 1960/1970s in Germany, treatment structures for drug addicts were implemented, separate from those existing for persons with AUD. Although public policy adopted a more integrated view of the use of psychotropic substances after the year 2000, separate treatment structures for alcohol and drugs largely remained in place. ${ }^{4}$

However, in Germany no empirical studies have been carried out on whether joint versus separate modalities of Rehab treatment for AUD and DUD patients might be associated with different treatment outcomes. When a German Rehab clinic for SUDs introduced a joint treatment for all types of SUDs, it was decided to examine whether this approach was associated with different treatment outcomes, compared to the customary separate treatment, as provided by a Rehab clinic with very similar characteristics in terms of location, sponsoring, staffing, funding, treatment philosophy, and treatment program characteristics. In addition, possible interactions between treatment modality and SUD pattern were examined. Premature treatment termination is considered as the primary outcome criterion. In addition, rates for lapse into substance use and treatment satisfaction were compared between types of treatment.

\section{Methods}

\section{Study settings}

Outcomes of patients admitted to the alcohol and drug dependence Rehab Unit of the LWL-Hospital Marsberg (joint treatment) were compared with those from the LWLHospital Warstein (separate treatment). Both clinics belong to the Landschaftsverband Westfalen-Lippe (LWL, Münster, Germany), a public organization providing psychiatric care, education, and cultural and social services within the German federal state North-Rhine Westphalia. The treatment facilities studied here were part of larger psychiatric hospitals where other mental disorders were being treated as well. Both clinics were located in the proximity of towns with a population of about 20,000 people, far away from larger cities.

Treatment was funded by the statutory pension insurance agencies, with its goal being the occupational reintegration of patients. Patients considered for admission to a Rehab Unit presented with a history of drug or alcohol dependence, had been detoxified prior to admission, and were at least 18 years old. Allocation of patients to the respective hospitals was not based on the individual's choice, but was carried out instead by the funding agencies, based upon free capacities. Inpatient Rehab of AUD is usually imbursed for a maximum of 16 weeks, for DUD it is 26 weeks; both can be extended, if necessary.

Treatment staff consisted of psychiatrists, clinical psychologists, social workers, occupational therapists, and nursing staff. The treatment program was in accordance with guidelines from the German societies of specialists in addiction treatment. ${ }^{5,6}$ It included the following: therapy planning; single and group therapy sessions; occupational therapy; family therapy; social skills training; health counseling; sporting activities; relaxation training; and recreational activities. With regard to the clinical profile of the individual patient, additional interventions could be offered, for example relapse prevention/recovery training, or treatment of comorbid conditions (eg, nicotine dependence, chronic anxiety, or posttraumatic stress disorder).

In the joint treatment facility, DUD and AUD patients were treated together. They were housed in the same buildings, were offered the same treatment package, and shared the same leisure facilities. Conversely, in the separate treatment facility, patients were located in different wards and did not attend the same therapeutic groups. Patients were accommodated in unlocked units. Leisure activities offered by the treatment facility were separated by AUD versus DUD treatment, but patients could come in mutual contact, especially during later stages of treatment, when they spent parts of their free time outside the clinic wards.

\section{Sampling}

All patients consecutively entering Rehab treatment during a 3-year period (2007-2009) were invited to provide written informed consent to participate in this prospective study. Patients were excluded if they were not able to give informed consent, for example due to serious cognitive 
deficits. Clinical categorization (AUD versus DUD) was carried out prior to entering the Rehab Unit by the referring funding agency. The funding agencies base their decisions on medical reports provided by previous treatment providers, for example, detoxification units.

\section{Measurements}

Shortly after admission, patients were interviewed by one of several trained research assistants, using a range of standardized questionnaires treatment motivation was measured using the German version of Texas Christian University Motivational Scale (TCU), a 29-item questionnaire assessing issues such as problem recognition, desire for help, treatment readiness, and external pressure to enter treatment. ${ }^{7,8}$ The Measurements in the Addictions for Triage and Evaluation interview (MATE) ${ }^{9}$ was performed, in order to gather data about sociodemographic characteristics, history of drug use and drug abuse treatment, and current legal situation. Contributors in the present study had been involved in the validation of the German versions of TCU (AB, FR, NS) or the MATE (AB), respectively, and they had therefore particular expertise with these instruments. Current SUDs and comorbid mental health conditions according to the diagnostic classification system ICD-10, chapter V (mental and behavioral disorders $)^{10}$ were routinely assessed at entry and during inpatient stay.

At discharge, patients were asked to complete the German version of the Client Satisfaction Questionnaire-8. The Client Satisfaction Questionnaire-8 is a unidimensional, 8 -item, questionnaire assessing patient satisfaction with treatment. ${ }^{11}$ It was selected because of satisfactory psychometric properties while are easily applicable as an add-on during the discharge process, due to its brevity. ${ }^{12}$

The primary outcome was here a premature termination of treatment, defined as a patient leaving against medical advice, or a patient being excluded from treatment on clinical or disciplinary grounds, or transfer to some other treatment.

Drug or alcohol use was repeatedly assessed by urine drug screenings or breath analysis, respectively, and by selfreports. Lack of drug/alcohol use during Rehab treatment was identified as a secondary outcome, although a single lapse into substance use did not necessarily result in exclusion from treatment on clinical/disciplinary grounds.

\section{Statistical analyses}

Patient characteristics (demographic and clinical variables, treatment motivation and satisfaction, treatment process, and outcome indicators) were compared between treatment modalities using $\chi^{2}$ tests and independent samples $t$-tests, or Welch-corrected $t$-tests in cases of inhomogeneous variances.

Multiple logistic regression analyses were performed for comparison of joint versus separate treatment modalities, with regard to premature termination and to lapse into substance use during treatment. The logistic regression models additionally included type of SUD (AUD, DUD, AUD + DUD), covariates associated with modality (gender, schooling completed, employment status, psychiatric comorbidity), and the interaction between SUD type and treatment modality.

\section{Power calculations}

We used the $g^{*}$ power software (Heinrich-Heine-Universität Düsseldorf, Düsseldorf, Germany) to determine the statistical power to identify an assumed effect of treatment modality in the logistic regression analysis..$^{13}$ The achieved sample size was $n=319$, and the empirical association of pretreatment characteristics with treatment modality (Nagelkerkes $R^{2}$ ) was 0.1 . We assumed a premature termination rate of $40 \%$ in separate treatment. An increase to $50 \%$ in joint treatment was then associated with a probability of 0.39 to be detected at a 5\% significance level (2-tailed), while an increase to 55\% was associated with a probability of 0.71 .

The study and its design were reviewed by the ethical scientific review board of the LWL Research Institute for Prevention and Mental Health (Director: Prof Dr G Juckel) and final approval was given. In addition, a scientific advisory board specifically established for this study approved study design and methods.

\section{Results Study sample}

A total of 334 patients were invited to participate to the study. Six declined the invitation to participate; hence, 328 patients were interviewed at baseline. Since 9 of these interviews were used to train the 3 research assistants and were excluded from statistical analyses, $\mathrm{n}=319$ patients effectively took part to the study, 174 in joint treatment (JT) and 145 in separate treatment (ST); 79 patients were treated for DUD, and 94 for AUD, in joint treatment; while 68 were treated for DUD and 77 for AUD in the separate treatment modality.

\section{Patient characteristics}

Regarding SUD profiles, an overlap was observed between alcohol- and drug-related disorders, respectively; $18.2 \%$ of the sample received concurrent diagnoses for both types of SUDs. Of those 58 patients concerned by this, $36.2 \%$ had 
received a diagnosis of an opiate dependence in addition to AUD, and $63.8 \%$ had received diagnoses related to cannabis and/or stimulants. The majority of the AUD + DUD group (60.3\%) had been admitted to Rehab with an indication of DUD treatment, with a nearly identical proportion in both modalities. The proportion of patients with AUD-only was $48.3 \%$, with DUD-only $33.5 \%$, including opiates $13.5 \%$, opiates and other illicit drugs $7.2 \%$, cannabis $3.8 \%$ cocaine/ stimulants $3.4 \%$, and cannabis plus stimulants/cocaine $5.6 \%$. As a consequence, 3 instead of 2 types of SUDs were included in the analysis: AUD-only, AUD plus DUD, and DUD-only. The 3 groups markedly differed with regard to age (AUD: mean 44.4 years [SD 8.5], AUD + DUD 29.7 [8.2], DUD 29.0 [6.7]), living together with a partner and/or children (AUD 30.6\%, AUD + DUD 21.3\%, DUD 12.6\%), migrant background (14.5\%/21.3\%/45.2\%), employment rate (43.8\%/23.0\%/29.1\%), and current legal problems such as being on probation or in mandatory treatment, or awaiting trial (10.1\%/39.7\%/54.2\%). In the AUD-only group, self-reported substance use other than alcohol was nearly 0 during the last 30 days before medical treatment. This group reported of a mean number of 20.1 days (SD 11.1) with alcohol use. The DUD group, in contrast, reported the consumption of alcohol (mean 4.7 days [SD 7.3]), cannabis (10.2 [13.2]), heroin (9.8 [12.9]), cocaine and other stimulants (3.7 [8.6]), and sedatives (3.4 [8.8]). The AUD + DUD group showed about the same frequency of alcohol use as the AUD-only group and about the same frequency of other substance use as the DUD group. The TCU motivation scales showed a higher problem recognition in the DUD group (mean 2.9 points [SD 0.7] on a scale ranging from 0 to 4), compared with the AUD group (2.1 [0.8]), and more perceived external pressure (1.9 [0.8] versus 0.9 [0.7]). The AUD + DUD group had results in between.

Comparison of patient characteristics between treatment facilities is presented in Table 1. Some marked differences between sites were observed. Females constituted 12.9\% of the sample in JT, compared with $6.8 \%$ in ST, where for logistical reasons only males were accepted into DUD treatment. Furthermore, JT patients had significantly more often completed school, were more frequently employed, and showed a lower rate of psychiatric comorbidity.

\section{Treatment process and outcome}

The indicators of treatment process (eg, possible presence of a lapse, days in treatment, days to regular treatment termination) and outcomes are shown in Table 2. Overall prevalence of premature termination and of regular completion was not statistically different between modalities. In addition,
Table I Sociodemographic and clinical characteristics

\begin{tabular}{|c|c|c|c|}
\hline Patient characteristics & $J T(n=174)$ & ST $(n=\mid 45)$ & $p$-values ${ }^{a}$ \\
\hline Age (mean, SD, range) & $\begin{array}{l}37.1(11.3) \\
18-67\end{array}$ & $\begin{array}{l}35.4(10.5) \\
18-64\end{array}$ & 0.17 \\
\hline Male & $87.1 \%$ & $93.2 \%$ & 0.046 \\
\hline Migrant background & $26.7 \%$ & $25.5 \%$ & 0.82 \\
\hline Stable partnership & $38.7 \%$ & $37.9 \%$ & 0.90 \\
\hline Living alone & $47.2 \%$ & $53.1 \%$ & 0.30 \\
\hline Living with partner/children & $25.8 \%$ & $19.3 \%$ & 0.18 \\
\hline Completed school & $95.1 \%$ & $86.2 \%$ & 0.006 \\
\hline Currently employed & $39.9 \%$ & $29.0 \%$ & 0.045 \\
\hline Current legal problems ${ }^{b}$ & $29.3 \%$ & $32.4 \%$ & 0.55 \\
\hline Illegal income last 30 days & $16.6 \%$ & $20.0 \%$ & 0.43 \\
\hline \multicolumn{4}{|l|}{ Treatment indication } \\
\hline AUD & $45.4 \%$ & $46.9 \%$ & 0.79 \\
\hline DUD & $54.6 \%$ & $53.1 \%$ & \\
\hline \multicolumn{4}{|l|}{ SUDs } \\
\hline Alcohol & $44.3 \%$ & $46.2 \%$ & 0.79 \\
\hline Drugs & $33.3 \%$ & $34.5 \%$ & \\
\hline Alcohol and drugs & $22.4 \%$ & $19.3 \%$ & \\
\hline Psychiatric comorbidity & $18.4 \%$ & $29.7 \%$ & 0.018 \\
\hline Current tobacco smoker & $90.8 \%$ & $92.4 \%$ & 0.061 \\
\hline \multicolumn{4}{|l|}{$\begin{array}{l}\text { psychotropic substance use, } \\
\text { mean (SD) }\end{array}$} \\
\hline $\begin{array}{l}\text { Months of regular use of } \\
\text { main substance, mean (SD) }\end{array}$ & I34 (III) & $124(104)$ & 0.39 \\
\hline $\begin{array}{l}\text { Number of substance } \\
\text { classes ever consumed,c }^{c} \\
\text { mean (SD) }\end{array}$ & $2.4(1.7)$ & $2.4(1.6)$ & 0.74 \\
\hline $\begin{array}{l}\text { Number. of substance } \\
\text { classes recently } \\
\text { consumed, },^{c, d} \text { mean (SD) }\end{array}$ & $1.8(1.3)$ & $2.0(1.4)$ & 0.29 \\
\hline Previous Rehab Treatment & $40.1 \%$ & $32.4 \%$ & 0.16 \\
\hline \multicolumn{4}{|c|}{ TCU motivation scales (range 0-4), mean (SD) } \\
\hline Problem recognition & $2.5(0.8)$ & $2.4(0.9)$ & 0.27 \\
\hline Desire for help & $3.0(0.6)$ & $3.0(0.8)$ & 0.71 \\
\hline Treatment readiness & $3.4(0.5)$ & $3.4(0.6)$ & 0.61 \\
\hline External pressure & I.3 (0.9) & $\mathrm{I} .4(0.9)$ & 0.64 \\
\hline
\end{tabular}

Notes: andependent samples $t$-tests $/$ Welch tests and $\chi^{2}$ tests. $p<0.05$ was considered as statistically significant: values shown in bold. ${ }^{\circ}$ Currently on probation, in mandatory treatment, or awaiting trial. 'Not including nicotine consumption. ${ }^{\circ}$ Last 30 days before detoxification treatment/rehabilitation treatment.

Abbreviations: AUD, alcohol use disorder; DUD, drug use disorder; JT, joint treatment; ST, separate treatment; SUD, substance use disorder; TCU, Texas Christian University.

patients with AUD-only showed a markedly lower premature termination rate $(28.9 \%)$ than those with DUD $(45.0 \%)$ or with DUD + AUD (49.5\%). The effect of SUD type was statistically significant ( $p=0.0003$ ).

The multiple logistic regression on premature treatment termination included treatment modality, type of SUDs (AUD, DUD, AUD + DUD), and treatment $\times$ disorder interactions as predictors, as well as control variable (gender, school completion, employment, and psychiatric comorbidity). Results show a statistically significant association of 
Table 2 Treatment process and treatment outcome

\begin{tabular}{|c|c|c|c|}
\hline & $\begin{array}{l}J T \\
(n=153)\end{array}$ & $\begin{array}{l}\text { ST } \\
(n=106)\end{array}$ & p-values ${ }^{\mathbf{a}}$ \\
\hline \multicolumn{4}{|l|}{ Treatment process } \\
\hline $\begin{array}{l}\text { At least I lapse during } \\
\text { treatment }\end{array}$ & $25.5 \%$ & $12.3 \%$ & 0.009 \\
\hline Days in treatment, mean (SD) & $90.5(47.8)$ & II $4.3(64.2)$ & 0.003 \\
\hline $\begin{array}{l}\text { Days until regular } \\
\text { completion, }{ }^{\mathrm{b}} \text { mean (SD) }\end{array}$ & $\begin{array}{l}114.3(37.9) \\
{[n=94]}\end{array}$ & $\begin{array}{l}|4| . \mid(43.6) \\
{[n=60]}\end{array}$ & 0.006 \\
\hline \multicolumn{4}{|l|}{ Treatment outcome } \\
\hline Premature termination & $37.9 \%$ & $44.1 \%$ & 0.26 \\
\hline Regular completion & $60.9 \%$ & $51.0 \%$ & 0.27 \\
\hline Transferred & $1.1 \%$ & $4.8 \%$ & 0.048 \\
\hline
\end{tabular}

Notes: ${ }^{a}$ Welch-corrected independent samples $t$-test for days in treatment and days until regular completion, Fisher's exact test for transferred, $\chi^{2}$ test for the other variables. $p<0.05$ was considered as statistically significant: values shown in bold. 'Includes only patients who regularly completed treatment.

Abbreviations: JT, joint treatment; ST, separate treatment.

Table 3 Multiple binary logistic regression analysis of premature termination of treatment

\begin{tabular}{|c|c|c|c|}
\hline Predictors & B & SE & $\begin{array}{l}\text { Adjusted odds } \\
\text { ratio }(95 \% \mathrm{Cl})^{\mathrm{a}}\end{array}$ \\
\hline ST (versus JT) & 0.34 & 0.37 & $1.41(0.69-2.90)$ \\
\hline \multicolumn{4}{|l|}{ Type of SUD (AUD as reference) } \\
\hline DUD & 0.86 & 0.38 & $2.36(1.12-4.98)$ \\
\hline AUD + DUD & 1.05 & 0.45 & $2.86(1.18-6.94)$ \\
\hline \multicolumn{4}{|l|}{ Treatment type $\times$ type of SUD } \\
\hline $\mathrm{ST} \times \mathrm{DUD}$ & -0.13 & 0.55 & $0.88(0.30-2.55)$ \\
\hline$S T \times A U D+A U D$ & -0.09 & 0.66 & $0.91(0.25-3.33)$ \\
\hline Male (versus female) & 0.21 & 0.44 & $1.23(0.52-2.93)$ \\
\hline $\begin{array}{l}\text { School completed (versus not } \\
\text { completed) }\end{array}$ & -0.29 & 0.41 & $0.75(0.33-1.68)$ \\
\hline Employed (versus unemployed) & -0.44 & 0.26 & $0.64(0.38-1.08)$ \\
\hline $\begin{array}{l}\text { Psychiatric comorbidity (versus no } \\
\text { psychiatric comorbidity) }\end{array}$ & -0.22 & 0.29 & $0.80(0.45-1.42)$ \\
\hline
\end{tabular}

SUD type (95\% confidence interval not including 1.00), but no significant association of treatment modality or any other of the covariates (Table 3). Exclusion of those cases with an undetermined outcome (eg, transfer to other treatment modalities) was associated with only minimal modification of the results (data not shown here).

Compared to AUD-only patients, probability of a lapse into substance use during treatment (Table 4) was significantly higher for DUD patients (odds ratio $=7.8$ ) and for DUD + AUD patients (odds ratio $=4.5$ ), respectively. Also, psychiatric comorbidity (most prevalent were depression, psychosis, anxiety disorders, and attention deficit/hyperactivity disorder) showed a statistically significant association with (lower) probability of at least 1 lapse, while there was no significant effect of treatment modality.
Table 4 Multiple binary logistic regression analysis of lapse into substance use during treatment

\begin{tabular}{|c|c|c|c|}
\hline Predictors & B & SE & $\begin{array}{l}\text { Adjusted odds } \\
\text { ratio }(95 \% \mathrm{Cl})^{\mathrm{a}}\end{array}$ \\
\hline ST (versus JT) & -0.56 & 0.72 & $0.57(0.14-2.34)$ \\
\hline \multicolumn{4}{|l|}{ Type of SUD (AUD as reference) } \\
\hline AUD & 2.06 & 0.51 & $7.82(2.89-21.17)$ \\
\hline AUD + DUD & 1.49 & 0.59 & $4.45(1.39-14.24)$ \\
\hline \multicolumn{4}{|l|}{ Treatment type $\times$ type of SUD } \\
\hline $\mathrm{ST} \times \mathrm{DUD}$ & -0.40 & 0.91 & $0.67(0.1 \mathrm{I}-4.0 \mathrm{I})$ \\
\hline $\mathrm{ST} \times \mathrm{AUD}+\mathrm{DUD}$ & -0.05 & 1.02 & $0.95(0.13-6.94)$ \\
\hline Male (versus female) & 0.37 & 0.82 & $1.44(0.29-7.19)$ \\
\hline $\begin{array}{l}\text { School completed (versus not } \\
\text { completed) }\end{array}$ & 0.14 & 0.64 & $1.15(0.33-4.02)$ \\
\hline Employed (versus unemployed) & -0.26 & 0.38 & $0.77(0.37-1.61)$ \\
\hline $\begin{array}{l}\text { Psychiatric comorbidity (versus } \\
\text { no psychiatric comorbidity) }\end{array}$ & -1.47 & 0.58 & $0.23(0.07-0.7 I)$ \\
\hline
\end{tabular}

Note: ${ }^{a} 95 \%$ Cls not including I indicate a statistically significant association: values shown in bold.

Abbreviations: AUD, alcohol use disorder; DUD, drug use disorder; JT, joint treatment; SE, standard error; ST, separate treatment; SUD, substance use disorder.

Satisfaction with treatment could be measured almost exclusively in patients who had regularly completed treatment (results not shown in the tables). The scale of the Client Satisfaction Questionnaire-8 items ranges from 1 (low satisfaction) to 4 (high satisfaction; some response scales had to be reversed first). In JT ( $n=81$ ), satisfaction averaged across items had a mean of 3.5 (SD 0.5), in ST ( $\mathrm{n}=51)$ it was 3.1 (SD $0.6)$. The difference was statistically significant $(p<0.001)$. Within each treatment modality, satisfaction levels were nearly identical for patients with AUD, DUD, or AUD + SUD.

\section{Discussion}

To the best of our knowledge, this is the first study which determined the difference in outcomes of a separate versus JT for patients with AUD and/or DUD. Treatment facilities were similar in terms of funding, setting, staffing, and treatment program philosophy.

Overall, treatment outcomes were comparable to those identified in previous German studies. ${ }^{14}$ In the current sample, about $51 \%$ of patients with a DUD or combined DUD/AUD, and about $29 \%$ of AUD patients, did not regularly complete treatment. Multiple logistic regression showed a statistically significant association of SUD type with premature treatment termination. In contrast, treatment modality and the SUD $\times$ modality interaction showed no significant association with premature termination.

Although the DUD and DUD + AUD groups were not homogenous (eg, stimulant and cannabis users were 5 years younger, on average, than the remaining DUD patients; cannabis users were less frequently on mandatory treatment/ current probation than the others), DUD patients are arguably 
perceived as part of a subculture with norms and behavioral standards which are barely compatible with those of the AUD patients, who used a substance which is legal and socially accepted. The AUD-only group in the present study was about 15 years older than the other patients, on average, they showed a low rate of legal problems, a comparably higher rate of employment, and lived alone less often. In addition, the proportion of patients with migrant background was lowest in this group. DUD patients indicated more problem recognition and reported higher external pressure than AUD patients on the TCU treatment motivation scales.

The implications of such differences for treatment process and treatment outcome are not immediately clear. They may perhaps facilitate the occurrence of conflicts. Such conflicts, in turn, may be associated with increasing levels of stress during adaptation to the therapeutic environment, and ultimately may have resulted in dysfunctional therapeutic group dynamics, therefore compromising the treatment success itself.

On the other hand, a JT approach may have some advantages. With this approach, AUD patients were possibly provided with additional knowledge relating to the mechanisms of addiction in general, beyond a focus on just alcohol. Conversely, those DUD patients who showed comorbid alcohol misuse may have learned that alcohol may be associated with severe medical consequences. ${ }^{15}$ And for those patients who are both affected by alcohol-related problems and illicit drugs, addressing both types of problems at the same time is facilitated.

While outcomes in the 2 treatment modalities appeared comparable, treatment completers in the JT group showed higher satisfaction with treatment than completers from the ST group. The mean difference was about $1 / 2$ a point on a 4-point scale. In fact, for some drug users, having been offered a JT modality may have contributed to a destigmatization of their disorder. It must be stressed, though, that treatment noncompleters could not be included here, and the possibility cannot be ruled out that noncompleters from the JT were more dissatisfied with treatment than those from ST.

A limitation of the study is its observational design, that is, patients were not randomly allocated to the different treatment conditions. This may well have introduced a range of unidentified biases, in addition to those possible confounders which were incorporated in the multiple regression analysis. Furthermore, although the different Rehab facilities were from the same treatment provider and were very similar with respect to staffing, treatment program, funding, or surrounding, it is still possible that clinics differed with respect to characteristics which were not addressed in the present study and could have an impact on treatment outcome, such as organizational climate or particular characteristics of the staff. Regarding assessment of patients' SUDs, the study used the diagnoses routinely made before treatment and within the treatment setting, that is, no additional diagnostic procedures were carried out. Reliability and validity of diagnoses were not verified and it can, therefore, not be ruled out that they were low. Finally, the statistical power to detect a clinically relevant effect was only moderate.

\section{Conclusion}

We found no evidence here for a difference between treatment modalities in terms of premature termination rates. The high prevalence of lapse into substance use observed in JT did not seem to have affected overall outcome levels. Conversely, the patients' satisfaction levels were higher in those who completed the JT modality. Further research should aim at identifying a range of parameters/clinical characteristics for the best possible allocation of patients to either a joint or a ST modality, so that premature termination rates, especially so in DUD clients, can be minimized.

\section{Acknowledgment}

We would like to thank Landschaftsverband Westfalen-Lippe for financing this study.

\section{Disclosure}

The authors report no conflicts of interest in this work.

\section{References}

1. Bergmark A. The relationship between alcohol and drug treatment systems. In: Klingemann H, Hunt G, editors. Drug Treatment Systems in an International Perspective: Drugs, Demons, and Delinquents. Thousand Oaks, CA, USA: Sage Publications; 2000:298-307.

2. MacGregor S. Drug treatment systems and policy frameworks: a comparative social policy perspective. Eur Addict Res. 1999;5:118-125.

3. Schott H. Das Alkoholproblem in der Medizingeschichte [The alcohol problem in the history of medicine]. Dtsch Arztebl. 2001; 98(30):A-1958-B-1962. German.

4. Muscat R, van de Mheen D, Barendregt C. Towards an integrated policy on psychoactive substances: a theoretical and empirical analysis. Strasbourg, France: Council of Europe; 2010. Available from: https:// rm.coe.int/1680745eac. Accessed April 24, 2018.

5. Geyer D, Batra A, Beutel M, et al. AWMF-Leitline: Postakutbehandlung alkoholbezogener Störungen. [AWMF-guideline: post-acute treatment of alcohol abuse and dependence]. Sucht. 2006;52(1):8-34. German.

6. Havemann-Reineke U, Küfner H, Schneider U, Günthner H, Schalast N, Vollmer HC. AWMF-Leitline: Postakutbehandlung bei Störungen durch Opioide [Guideline: post-acute treatment of opiate abuse and dependence]. Sucht. 2004;50(4):226-257. German.

7. De Weert-Van Oene GH, Schippers GM, De Jong CA, Schrijvers GA. Motivation for treatment in substance-dependent patients. Psychometric evaluation of the TCU motivation for treatment scales. Eur Addict Res. $2002 ; 8: 2-9$ 
8. Buchholz A, Glöckner-Rist A, Scherbaum N, Rist F. Die Texas Christian University - Motivational Scales in deutscher Sprache: TCU-MS-d [The Texas Christian University Motivational Scales in German language: TCU-MS-d]. In: Glöckner-Rist A, editor. Zusammenstellung sozialwissenschaftlicher Items und Skalen. ZIS Version 14.00 [Compilation of items and scales from the social sciences]. Bonn, Germany: GESIS; 2010. German.

9. Schippers GM, Broekman TG. MATE, Measurements in the Addictions for Triage and Evaluation. Development of an Instrument Assessing Patient Characteristics in Substance Abuse Treatment. Final Report. Amsterdam, Netherlands: AIAR, Department of Psychiatry, Academic Medical Center, University of Amsterdam, and Nijmegen, the Netherlands: Bureau Bêta; 2007. Available from: http://www.mateinfo. eu/pubs/31000068.pdf. Accessed April 24, 2018.

10. World Health Organization. ICD-10: International Statistical Classification of Diseases and Related Health Problems. 10th revision, 2nd ed. Geneva: World Health Organization; 2004.
11. Attkisson CC, Zwick R. The client satisfaction questionnaire. Psychometric properties and correlations with service utilization and psychotherapy outcome. Eval Program Plann. 1982;5(3):233-237.

12. Schmidt J, Lamprecht F, Wittmann WW. Zufriedenheit mit der stationären Versorgung. Entwicklung eines Fragebogens und erste Validitätsuntersuchungen. [In-patient treatment satisfaction. Development and first validation of a questionnaire]. Psychother Med Psychol. 1989;39(7):248-255. German.

13. Faul F, Erdfelder E, Buchner A, Lang AG. Statistical power analyses using $\mathrm{G}^{*}$ Power 3.1: Tests for correlation and regression analyses. Behav Res Methods. 2009;41(4):1149-1160.

14. Statistik der Deutschen Rentenversicherung: Rehabilitation [Statistics from the German age insurance: rehabilitation]. Berlin, Germany: Deutsche Rentenversicherung Bund; 2014. German.

15. Staiger PK, Richardson B, Long CM, Carr V, Marlatt GA. Overlooked and underestimated? Problematic alcohol use in clients recovering from drug dependence. Addiction. 2013;108(7):1188-1193.
Substance Abuse and Rehabilitation

\section{Publish your work in this journal}

Substance Abuse and Rehabilitation is an international, peer-reviewed, open access journal publishing original research, case reports, editorials, reviews and commentaries on all areas of addiction and substance abuse and options for treatment and rehabilitation. The manuscript management system is completely online and includes a very quick and fair

\section{Dovepress}

peer-review system. Visit http://www.dovepress.com/testimonials.php to read real quotes from published authors. 